\title{
Microstructural and mechanical characterization of carbon aerogels: An in- situ and digital image correlation-based study
}

\author{
Ameya Rege $^{\mathrm{a}, *}$, Marina Schwan ${ }^{\mathrm{a}, *}$, Liudmila Chernova ${ }^{\mathrm{a}}$, Markus Hillgärtner ${ }^{\mathrm{b}}$, Mikhail Itskov ${ }^{\mathrm{b}}$, \\ Barbara Milow ${ }^{\mathrm{a}}$ \\ anstitute of Materials Research, German Aerospace Center, Linder Höhe, 51147 Cologne, Germany \\ ${ }^{\mathrm{b}}$ Department of Continuum Mechanics, RWTH Aachen University, Kackertstraße 9, 52072 Aachen, Germany
}

\section{A R T I C L E I N F O}

\section{Keywords:}

Carbon aerogels

In-situ scanning electron microscopy

Digital image correlation

Uniaxial tests

Three-point bending tests

\begin{abstract}
A B S T R A C T
The mechanical behavior of carbon aerogels is not very well understood, presenting a bottle-neck in synthesizing aerogels for specific applications where mechanical loads play a role. Accordingly, in this paper, three different types of carbon aerogels with varying mechanical properties and flexibility are synthesized and analyzed. The morphology is characterized by using a scanning electron microscope (SEM) and nitrogen adsorption-desorption isotherms. The mechanical behavior is investigated under uniaxial quasistatic compression as well as in-situ compression under a SEM. While in-situ tests reveal microstructural evolution under deformation, the macroscopic deformation is described by the digital image correlation. Based on cyclic compression tests with stepwise increasing strain amplitude, novel empirical relations are proposed to describe the damage characteristics such as energy dissipation and residual deformation. Furthermore, while testing carbon aerogels under tension is not very feasible, three-point bending tests are conducted and the resulting flexural properties of carbon aerogels are identified.
\end{abstract}

\section{Introduction}

Carbon aerogels (CA) are open-porous materials with a three-dimensional micro- and mesoporous network which was first described by Richard W. Pekala [1]. They are typically obtained by carbonization of organic aerogels under an inert atmosphere [2,3]. Due to many interesting properties, such as a large surface area, a tunable high porosity, and a low bulk density, carbon aerogels are promising materials for hydrogen storage or as a radiation adsorption material [4,5]. Combined with their high electrical conductivity, they are ideally suited for electrochemical double layer capacitors or as cathode material for lithium-sulfur batteries [6-10].

The mechanical properties of carbon aerogels play a significant role, for instance, in foundry applications for adsorption of foundry gases [11]. These have so far not been investigated extensively in the literature. Reports on the mechanical behavior and properties of aerogels, in general, are getting increasing attention within the aerogel community. There have been quite significant studies describing the mechanical behavior of silica [12-15] and organic [16,17] aerogels. With deeper understanding of the materials behavior, constitutive models can be developed for prediction of the mechanical properties based on either empirical or, of more interest, micromechanical parameters. Such models are indispensable for designing products tailored for specific applications. However, for models to be developed, an in-depth understanding of the morphology, its evolution under deformation and the mechanical behavior is necessary. As a first step to analyze the mechanical response of aerogels, uniaxial quasistatic compression/ tension tests are often conducted. Generally, compression tests are done by preparing cuboidal or cylindrical specimens and compressing them uniaxially in a Universal Testing Machine (UTM). Furthermore, cyclic tests with step-wise increasing strain amplitude reveal many inelastic features. In contrast, testing aerogels under tension is very difficult. First, it is hard to prepare dog-bone shaped specimens of aerogels (notwithstanding these difficulties, cellulose aerogels have been successfully tested under tension [18]). Second and most important, aerogel specimens tend to break at the specimen gripping either while setting the experiment up, or much before the anticipated actual failure strain is reached. This behavior of aerogels is primarily attributed to their extreme brittle nature. In such cases, literature recommends the use of flexural tests, or colloquially known as the three-point bending test. The sample preparation and setup of this experiment are very easy and similar to compression tests.

\footnotetext{
* Corresponding authors.

E-mail addresses: ameya.rege@dlr.de (A. Rege), marina.schwan@dlr.de (M. Schwan).
} 
With improved imaging techniques, deformation mapping of the specimen surfaces is easily done by means of the well-known digital image correlation (DIC) procedure. While using a single camera-based optical measuring, cuboidal specimens are preferred over the cylindrical ones because the optical measuring system, tracks a 2-d image surface and cannot capture points on a 3-d curved surface. DIC is an optical technique for performing displacement and strain measurements. It uses the principles of photogrammetry, i.e. determining the exact positions of surface points by comparing stereological series of digital photographs captured at different stages of deformation. Although it is effectively used on metals, rubbers, etc., it is very hard to implement on aerogels. This is primarily because of surface irregularities, which makes it difficult to spray on it. Moreover, due to this irregular powdery surface, it is troublesome for the optical measuring system to track these sprayed points.

While DIC informs about the macroscopic deformation of a specimen, in-situ scanning electron microscopy (SEM) reveals the evolution of the microstructure under deformation. With the help of in-situ SEM compression tests, the mechanical behavior of the porous aerogel network can be visualized. Such experiments have been performed under an SEM beam allowing for a novel in-situ analysis of carbon aerogels, and are useful to identify deformation behavior and damage characteristics. The in-situ SEM investigation allows also for an edge-on view of crack growth.

The objective of this paper is to experimentally identify the microscale damage processes under loading-unloading condition, through insitu and SEM analysis and their correlation with digital image correlation results. To the best of our knowledge, results of such tests on ductile and stiff carbon aerogels at this scale have not been published previously. Operating at this scale allows reproducing deformations mechanisms from full scale tests at a lower material cost than those available through conventional tests. Also high magnifications under an SEM beam are possible. Moreover, the mechanical behavior of carbon aerogels has not been studied using these above-mentioned procedures. The goal of this work is thus to shed light on the micro- and macromechanics of carbon aerogels, in a way that would enhance its applicability and further modeling studies. For our study, we produced three types of carbon aerogels. The first type is termed Pekala-carbon aerogel (p-CA), its synthesis route is based on a recipe developed by Pekala [1]. It is a ductile aerogel with a nanostructured network. The second type is stiff carbon aerogel (s-CA) synthesized according to Saliger et al. [19]. Its structure consists of relatively large particles of about $2 \mu \mathrm{m}$ and also large pores. A third type is super-flexible carbon aerogel (sf-CA), its synthesis is based on the work by Schwan and Ratke [20]. This aerogel is very soft compared to p-CA and s-CA, and can be reversibly deformed up to $30 \%$ compression.

The paper is organized as follows. Section 2 reviews the synthesis of the three types of carbon aerogels in consideration. Furthermore, it discusses the tools used to characterize these aerogels, by means of scanning electron microscopy, in-situ SEM, uniaxial compression, digital image correlation and flexural tests. Section 3 illustrates the results from all the characterization and mechanical tests.

\section{Materials and methods}

\subsection{Synthesis of carbon aerogels}

In the first step, resorcinol-formaldehyde (RF) aerogels (p-RF; s-RF and sf-RF) were synthesized. At room temperature, resorcinol (R) was dissolved in deionized water (W) and stirred at $150 \mathrm{rpm}$ using a crossmagnetic stirring bar. Then, an aqueous solution of formaldehyde (F) and solid sodium carbonate (Cat) were added subsequently to the stirred resorcinol solution (see Table 1). After $5 \mathrm{~min}$, the $\mathrm{pH}$ of sf-RF solution was adjusted to 5.4-5.6 by dropwise addition of $2 \mathrm{~N}$ nitric acid solution, the $\mathrm{pH}$ of s-RF and $\mathrm{p}-\mathrm{RF}$ solutions stayed unmodified. The stirring at room temperature was continued, and the homogeneous transparent solution was placed in a sealable polypropylene container for seven days in an oven at $60^{\circ} \mathrm{C}$. During gelation and curing, the $\mathrm{RF}$ solutions became brown or beige. After seven days of gelation and aging, the wet s-RF gels were dried for one day at $80^{\circ} \mathrm{C}$ in a drying cabinet at ambient pressure. The sf-RF and p-RF gels of about $100 \mathrm{~mL}$ volume were cooled down to the room temperature and transferred into an acetone bath of about $300 \mathrm{~mL}$ in order to remove residual reagents and to exchange water by acetone being soluble in supercritical carbon dioxide. The bath was not stirred or shacked. The acetone washing was repeated six times within three days. The supercritical drying was carried out with $\mathrm{CO}_{2}$ in an autoclave of $12 \mathrm{~L}$ volume (Eurotechnica, Germany) at $50^{\circ} \mathrm{C}$ and $10 \mathrm{MPa}$ for approximately $32 \mathrm{~h}$. The degassing rate was adjusted to $0.01 \mathrm{MPa} \min ^{-1}$.

In the second step, the resulting RF aerogels were carbonized. The aerogels were placed in the furnace, purged three times with argon, and heated to the carbonization temperature of $1000^{\circ} \mathrm{C}$. The heat rate was adjusted to $6-7 \mathrm{~K} \mathrm{~min}^{-1}$, and argon was passed through the furnace under a pressure of $4 \mathrm{bar}$. The samples were maintained at these conditions for $60 \mathrm{~min}$ and then cooled down to room temperature under a flow of argon.

\subsection{Microstructural characterization}

The envelope density of sf-CA was calculated from measured volume and weight of an aerogel piece. The envelope density of p-CA and s-CA were measured with GeoPyc (Micromeritics). The skeletal density was measured with AccuPyc (Micromeritics). The surface area and pore size distribution of aerogels were determined by the nitrogen adsorption-desorption tests using the Brunauer-Emmet-Teller (BET)/ BarretJoyner-Halenda (BJH) methods and by carbon dioxide adsorption method NLDFT for microporosity (TriStarII, Micromeritics, Germany). Before analysis, the samples were outgassed for $24 \mathrm{~h}$ at $200^{\circ} \mathrm{C}$ and $10 \mathrm{~Pa}$.

The microstructure of the aerogels was investigated by performing SEM using a ZEISS ULTRA55 scanning electron microscope (Zeiss SMT, Oberkochen, Germany) equipped with a field emission gun.

The in-situ compression tests were performed with carbon aerogels cut to approximately $10 \mathrm{~mm} \times 10 \mathrm{~mm} \times 5 \mathrm{~mm}$ samples and a rate of compression of $0.5 \mu \mathrm{m}$ per second. The measurements were carried out by means of a Kamrath \& Weiss Deformation Devices System (DDS) (Fig. 1a). The micro tester was operated inside the chamber of a

Table 1

Synthesis parameters of carbon aerogels.

\begin{tabular}{|c|c|c|c|c|c|c|}
\hline Type of aerogel & $\mathrm{R}:$ Cat molar ratio & $\begin{array}{l}\mathrm{R}: \mathrm{W} \text { molar } \\
\text { ratio }\end{array}$ & $\begin{array}{l}\mathrm{R}: \mathrm{F} \text { molar } \\
\text { ratio }\end{array}$ & Stirring time, min & Drying conditions & Pyrolysis conditions \\
\hline $\mathrm{p}-\mathrm{CA}$ & 200 & 0.019 & 0.5 & 30 & With supercritical $\mathrm{CO}_{2}$ & \\
\hline s-CA & 1500 & 0.044 & 0.74 & 30 & Ambient pressure, $80^{\circ} \mathrm{C}, 1$ day & $1000^{\circ} \mathrm{C}$, one hour with Argon \\
\hline sf-CA & 50 & 0.008 & 0.5 & 60 & With supercritical $\mathrm{CO}_{2}$ & \\
\hline
\end{tabular}


a

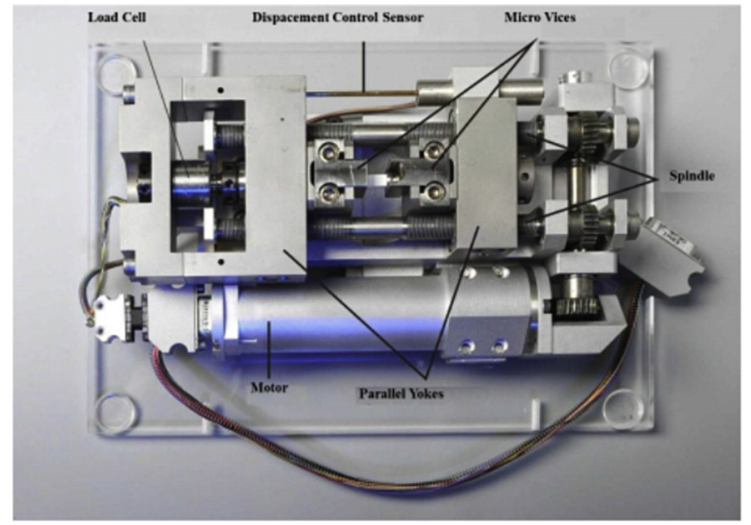

C

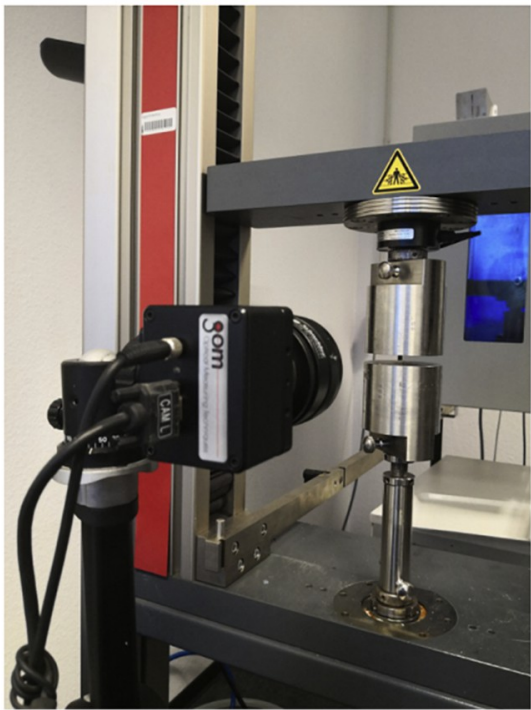

b

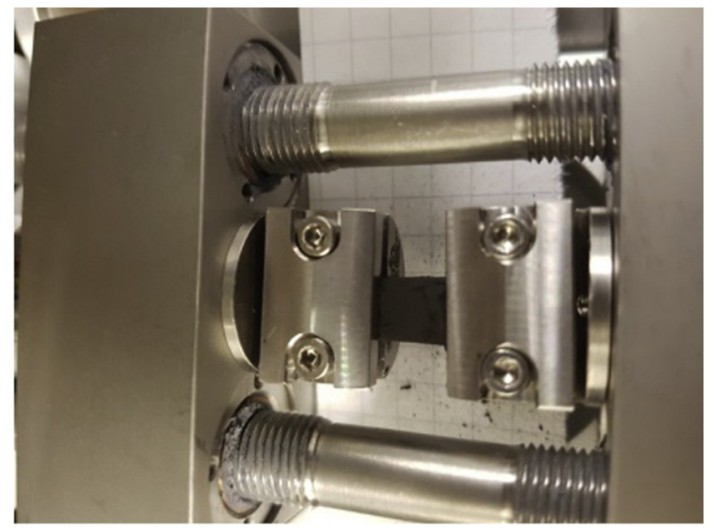

d

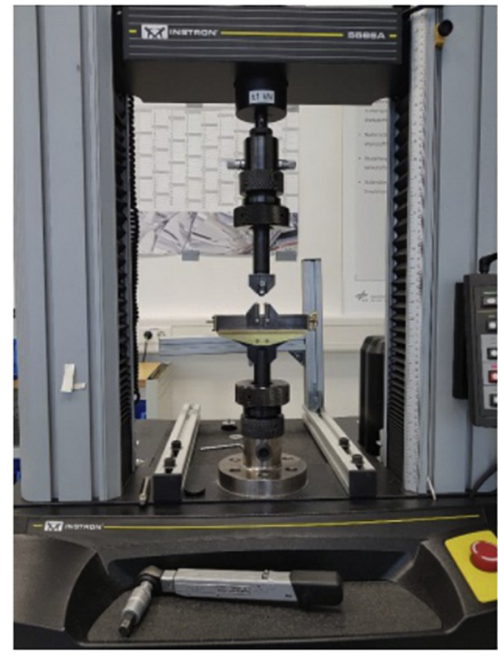

Fig. 1. a) Deformation Devices System (DDS) for in-situ investigations, b) s-CA sample placed in Deformation Devices System, c) test setup for the uniaxial testing along with the camera used for DIC, d) test setup for the three-point bending test.

scanning electron microscope. An aerogel sample (s-CA) chosen for the experimental testing and the sample position in the micro tester are shown in Fig. $1 \mathrm{~b}$.

\subsection{Uniaxial quasistatic compression}

All carbon aerogel specimens were tested on the UTM Z10 by Zwick/Roell, Germany with a load cell of $1 \mathrm{kN}$ (see Fig. 1c). The tests were performed at a strain rate of $0.1 \% \mathrm{~s}^{-1}$ as specified by Pekala et al. [21]. Two types of tests were conducted. First, all aerogels under consideration were compressed up to brittle fracture. Accordingly, the fracture strain was identified. Then, step-wise cyclic compression was applied with a maximal strain slightly below the fracture strain. This second set of tests provides information regarding the inelastic effects, such as stress softening and residual strains. Displacements were measured online by a contact-less DIC measuring system by Gesellschaft für optische Messtechnik (GOM), Germany. All the tests were conducted at room temperature and at ambient humidity. The specimens were tested as prepared without further conditioning.

\subsection{Digital image correlation}

At first, a stochastic pattern of white spray paint was put on the sample surface to be measured. A digital camera (see Fig. 1c) was used to capture the images of the specimen under sufficient illumination. An image capturing frequency of $6-12 \mathrm{~Hz}$ (depending on the sample) was used in the experiment. By comparing the grey scale values in the so obtained image series, the relative displacement of each pixel was computed. The stochastic pattern on the surface provides a good gradient in the grey scale values of neighboring pixels, thus resulting in the more accurate measurement. The relative displacement values serve as a basis for the further calculation of strains by the software ARAMIS v6.3.0.

A rectangular cross-sectional region was used for computation, omitting the surface edges (given their irregularities). ARAMIS observes the deformation of the region of interest with the help of many small square areas of the image, also known as facets. Carbon aerogel specimens have a very rough and powdery surface. The mapping function in DIC uses contrast markers (sprayed paint pattern) to correlate successive images. Care was taken to mark aerogels with a sprayed paint, such that it adheres to the specimen surface and does not fall off immediately, when wiped or at the onset of deformation. However, when powdery chunks fall from the specimen surface for instance due to cracks, it is no longer possible to track the displacements and strains in that particular region. Accordingly, multiple tests for every type of aerogel were conducted until the region of interest was satisfactorily covered in the marked pattern.

\subsection{Flexural testing}

The three-point bending tests were performed on a UTM (Instron, Germany) with a $1 \mathrm{kN}$ loading cell (see Fig. 1d). The loading velocity was adjusted to $0.4 \mathrm{~mm} \mathrm{~min}^{-1}$. The samples were cut with scalpel to bar-shaped specimens. 


\section{Results and discussion}

The general properties such as density, porosity and specific surface area are summarized in Table 2.

The microstructure of carbon aerogels is shown in Fig. 2 a-c. Fig. 2a depicts the microstructure of a p-CA sample. It consists of very small particles of about $10-20 \mathrm{~nm}$. Due to a high amount of catalyst used for synthesis, the addition reaction was prolonged and led to the formation of small particles. The nitrogen adsorption-desorption methods show that p-CA has the highest surface area due to the smallest size of particles and the highest mesopore volume of about $0.85 \mathrm{~cm}^{3} \mathrm{~g}^{-1}$. The macropore volume is $2.12 \mathrm{~cm}^{3} \mathrm{~g}^{-1}$. From a broad pore size distribution, we see two peaks in the mesoporous region, the first peak at $2-3 \mathrm{~nm}$ and

Table 2

General properties of carbon aerogels.

\begin{tabular}{llll}
\hline Properties & p-CA & s-CA & sf-CA \\
\hline Skeletal density, $\mathrm{g} \mathrm{cm}^{-3}$ & 2.16 & 2.23 & 2.72 \\
Envelope density, $\mathrm{g} \mathrm{cm}^{-3}$ & 0.29 & 0.23 & 0.04 \\
Porosity, \% & 87 & 87 & 98 \\
Macropore volume, $\mathrm{cm}^{3} \mathrm{~g}^{-1}$ & 2.12 & 3.60 & 17.83 \\
Mesopore volume (BJH), $\mathrm{cm}^{3} \mathrm{~g}^{-1}$ & 0.85 & 0.01 & 0.04 \\
Micropore volume (t-plot), $\mathrm{cm}^{3} \mathrm{~g}^{-1}$ & 0.12 & 0.20 & 0.18 \\
Specific surface area (BET), $\mathrm{m}^{2} \mathrm{~g}^{-1}$ & 688 & 624 & 561 \\
Micropore area (t-plot), $\mathrm{m}^{2} \mathrm{~g}^{-1}$ & 286 & 521 & 464 \\
External surface area (t-plot), $\mathrm{m}^{2} \mathrm{~g}^{-1}$ & 402 & 103 & 97 \\
\hline
\end{tabular}

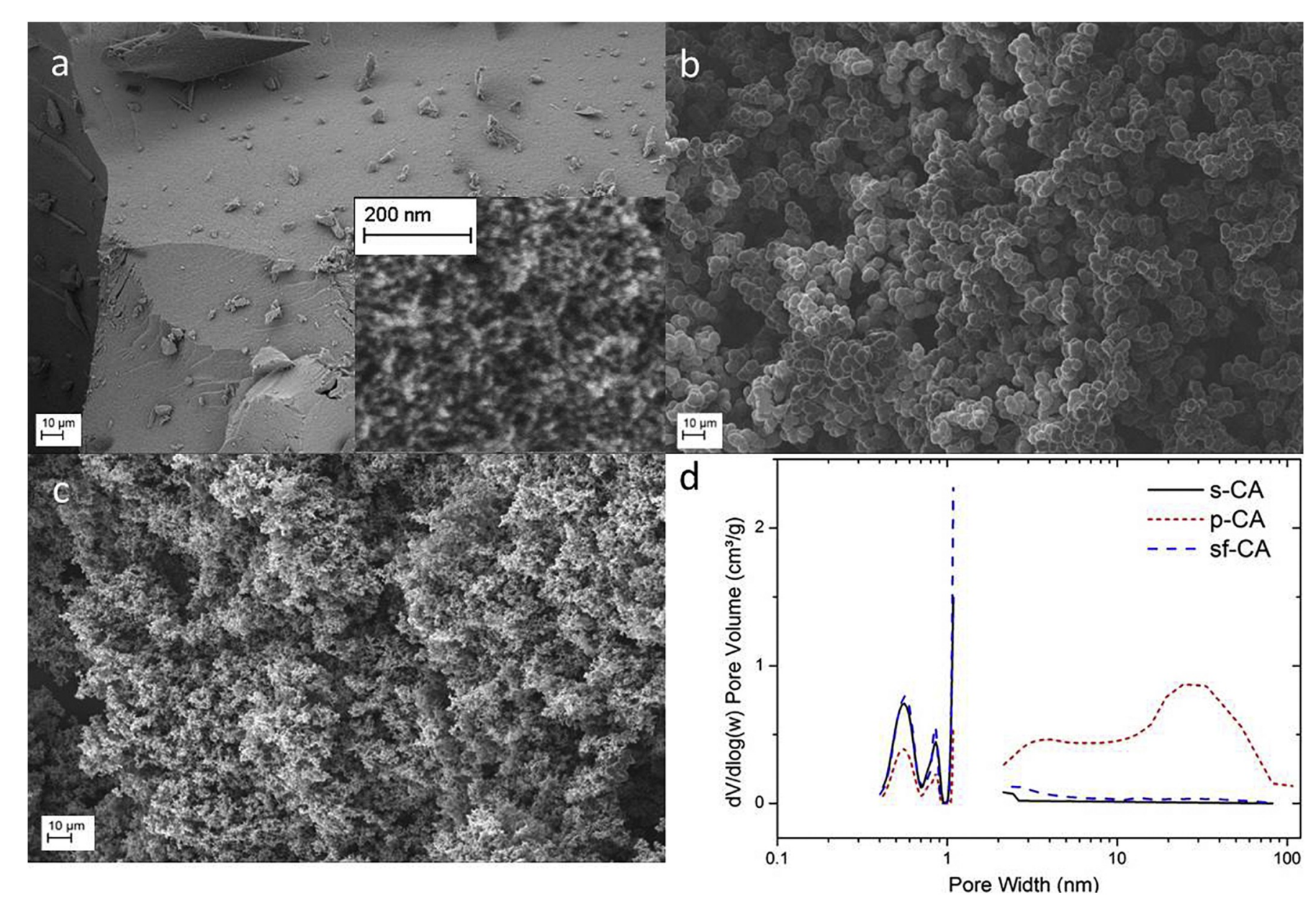

the second at $20-30 \mathrm{~nm}$ (Fig. 2d). The micropores are also divided in two main fields: $0.5-0.6$ and $0.8-0.9 \mathrm{~nm}$. This carbon aerogel exhibits the highest envelope density of about $0.29 \mathrm{~g} \mathrm{~cm}^{-3}$ which could be explained by the high shrinkage (about 6-7\%) during supercritical drying. By means of supercritical drying, the preservation of wet gel structure and shape is not always guaranteed. Shrinkage could be avoided by very high $\mathrm{R} / \mathrm{C}$ and $\mathrm{R} / \mathrm{W}$ molar ratios. In our case, the R/W ratio used was quite low, which led to shrinkage during drying. Another factor which also could lead to shrinkage is solvent exchange. The solvent exchange step could have remained incomplete and some water amount could penetrate inside of the gel. Especially in micropores and small mesopores detected by nitrogen sorption, the diffusion of the solvent occurs very slowly. Residual water could cause surface tension and fractures in fragile network. Prolonged washing time could improve the producing of less shrunk aerogels. Additional high radial volume shrinkage of $28 \%$ occurred during carbonization. Due to small polymer nodules this kind of aerogels is very sensitive to sintering during carbonization.

The network of s-CA xerogel shown in Fig. 2b consists of much larger particles compared to $\mathrm{p}-C A$. The particles exhibit a similar shape but are quite bigger, about $5-10 \mu \mathrm{m}$ in diameter. This is a result of the small amount of catalyst used which led to a prolonged condensation step during synthesis. Most of the particles are interconnected with wide necks which can increase the strength of xerogels. The pore size distribution of s-CA is very broad from $0.5-0.6 \mathrm{~nm}$ (Fig. 2d) in the micropore region to $20-40 \mu \mathrm{m}$ in the macropore region. The macropore volume is $3.60 \mathrm{~cm}^{3} \mathrm{~g}^{-1}$ and is comparable with $\mathrm{p}$-CA. In the mesopore region, only a low pore volume of about $0.01 \mathrm{~cm}^{3} \mathrm{~g}^{-1}$ could be found.

Fig. 2. (a-c) SEM images with microstructures of (a) p-CA, (b) s-CA, (c) sf-CA, (d) pore size distribution shows strong differences in the microstructure of carbon aerogels. 
Due to a high resorcinol amount, the bulk density of s-CA is very high $\left(0.23 \mathrm{~g} \mathrm{~cm}^{-3}\right)$, which is expected and well known for xerogels. During drying no shrinkage of RF gel could be observed. This is due to low capillary forces in large pores and quite thick pore walls which could withstand surface tension. After carbonization s-CA xerogel shrunk about $19 \%$.

The flexible carbon aerogel (sf-CA), since it is produced with high amount of sodium carbonate as a catalyst, shows smaller particles than the s-CA. In the SEM image illustrated in Fig. 2c, particles of about $1 \mu \mathrm{m}$ and relatively large macropores about $10-30 \mu \mathrm{m}$ can be observed. This is due to the base-acid synthesis route of flexible carbon aerogel. In addition, this aerogel exhibits a high micropore volume of $0.18 \mathrm{~cm}^{3}$ $\mathrm{g}^{-1}$, and similar to $\mathrm{s}-\mathrm{RF}$, a low mesopore volume of $0.04 \mathrm{~cm}^{3} \mathrm{~g}^{-1}$. The macropore volume is much higher than p-CA and s-CA $\left(17.83 \mathrm{~cm}^{3} \mathrm{~g}^{-1}\right)$. sf-CA exhibits the highest porosity of $98 \%$ and the lowest envelope density caused by lowest resorcinol amount. This aerogel did not show some shrinkage after drying, although the aerogel exhibits high micropore volume. The carbonization caused radial shrinkage of about $18 \%$.

In-situ compression tests allow an insight to the deformation behavior of carbon aerogels. By this means, the deformation of pore walls and the fracture in the network under loading can be observed. Additionally, the effect of pore-to-particle size ratio on the deformation behavior under uniaxial loading could be estimated. Nevertheless, the method has some limitations, e.g. non-conductive samples or networks consisting of very small particles $(<50 \mathrm{~nm}$ like p-CA) cannot be analyzed. Due to these limitations, p-CA could not be tested with this technique.

For stiff carbon aerogel, a represented position with small and large pores was chosen (Fig. 3a). The largest pore was tracked during test.
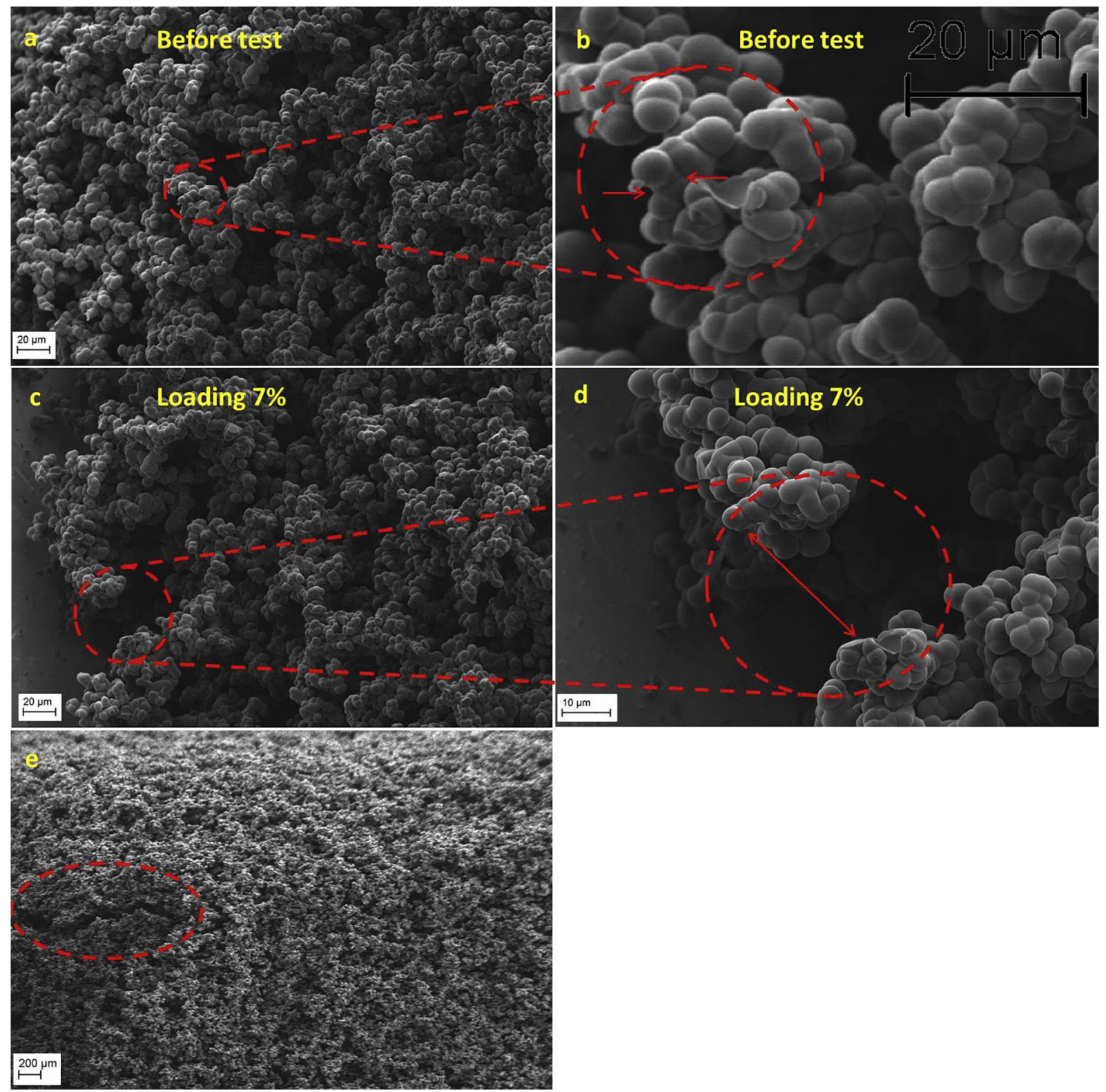

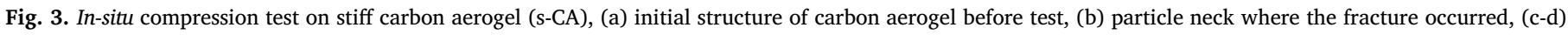
fracture in the network between particles, (e) crack formation under loading of the stiff carbon aerogel. 

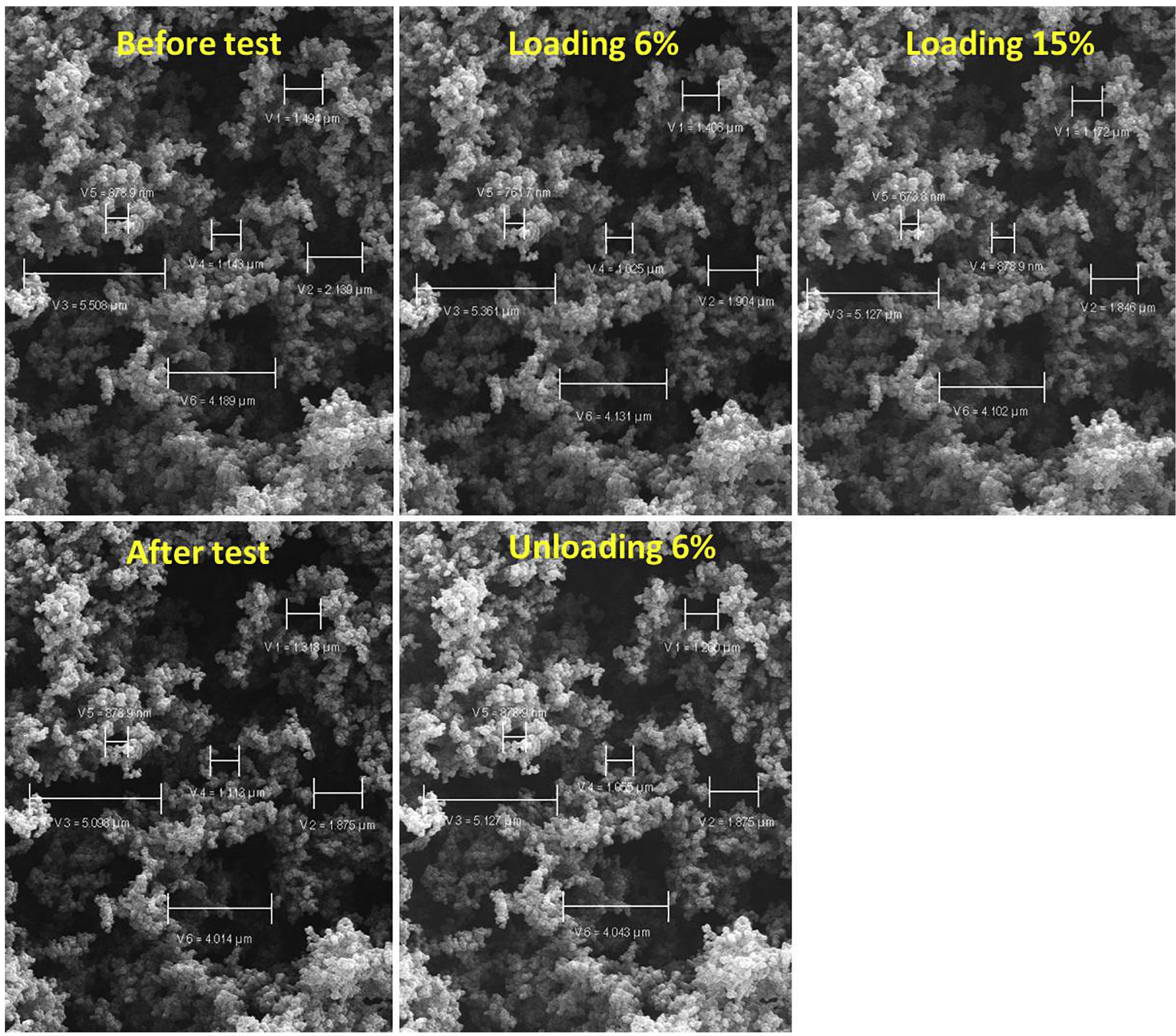

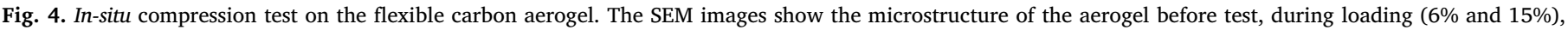
unloading and after test. Six pores with different pore sizes were marked (V1-V6), their sizes are given after each step of the compression test.

One particle neck in the chosen pore wall before loading is shown in Fig. 3b and marked with arrows. After the compression of $7 \%$ we can see a fracture in the pore wall. Under critical loading, the particles disconnect and the pore opens. As expected, the fracture takes place between the particles. The neck represents a predetermined breaking point due to the lower moment of inertia at this position. The in-situ test clearly confirms the theory. The arrows in Figs. 3c-d show the fracture surfaces of disconnected particles. No other changes or deformations in the network are visible (Figs. 3c-d). The size of other smaller pores is the same.

As shown in Fig. 3e a large crack is formed in the sample. Based on our previous experience with s-CA compression tests, we assume that the crack was formed earlier, at lower compression. The deformation behavior of flexible carbon aerogels differs very strongly compared to stiff aerogels. For analysis, we selected six pores and named them V1 to
V6 (Fig. 4). These pores could be divided in three groups: first, V4 and V5 are the smallest pores with pore width of about $1 \mu \mathrm{m}(1.14 \mu \mathrm{m}$ and $0.88 \mu \mathrm{m}$ respectively); to the second group belong pores V1 and V2 which are of about $1.5-2 \mu \mathrm{m}$, and the third group consists of the largest pores with a pore width of about $4-5.5 \mu \mathrm{m}$.

\subsection{Deformation of small pores V4, V5}

The smallest pores underwent the highest deformations. At $15 \%$ of compression, pore V4 was deformed to about $23 \%$ and V5 to $24 \%$. The smallest pores recovered nearly completely after unloading. While the irreversible deformation of pore V4 is $2.6 \%$, pore V5 exhibited no irreversible deformation (see Table 3 and Fig. 5). 
Table 3

Deformation of pores V1-V6 during loading and unloading of flexible carbon aerogel.

\begin{tabular}{lllllll}
\hline Pore & $\mathrm{V} 1, \mu \mathrm{m}$ & $\mathrm{V} 2, \mu \mathrm{m}$ & $\mathrm{V} 3, \mu \mathrm{m}$ & $\mathrm{V} 4, \mu \mathrm{m}$ & $\mathrm{V} 5, \mu \mathrm{m}$ & $\mathrm{V} 6, \mu \mathrm{m}$ \\
\hline Before test & 1.49 & 2.14 & 5.51 & 1.14 & 0.88 & 4.19 \\
Loading 6\% & 1.41 & 1.90 & 5.36 & 1.02 & 0.76 & 4.13 \\
Loading 15\% & 1.17 & 1.85 & 5.13 & 0.88 & 0.67 & 4.10 \\
Unloading 6\% & 1.26 & 1.87 & 5.13 & 1.05 & 0.88 & 4.01 \\
After test & 1.32 & 1.87 & 5.10 & 1.11 & 0.88 & 4.01 \\
\hline
\end{tabular}

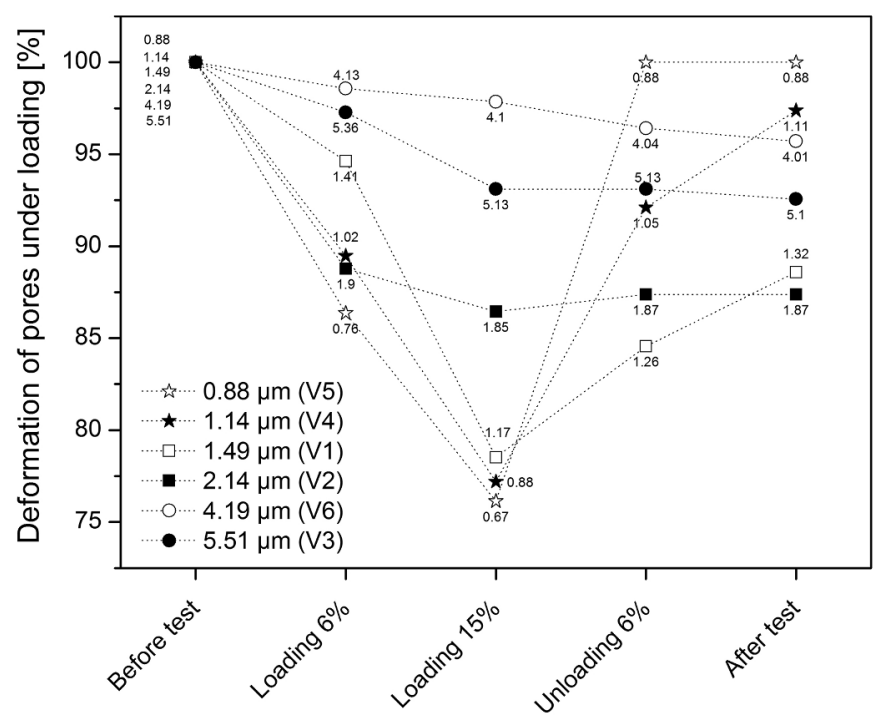

Fig. 5. Deformation of pores under loading based on in-situ SEM measurements. The smallest pores (V4 and V5) undergo the highest deformation and recover after test almost completely back. The middle large pores (V1 and V2) undergo lower deformation, their part on irreversible deformation is higher compared with smaller pores. Large pores (V3 and V6) deform slightly and completely irreversible. The numbers are given in $\mu \mathrm{m}$.

\subsection{Deformation of middle large pores V1, V2}

In the reference state, the pore size of V1 was about $1.49 \mu \mathrm{m}$ and V2 about $2.14 \mu \mathrm{m}$. After loading to $15 \%$, the deformation of these pores reached $21.5 \%$ and $13.5 \%$, respectively. Here we again see similar behavior, the smaller the pore, the higher the deformation. After unloading the smaller pore V1 recovered to $87 \%$ of the initial size and the larger pore V2 to $88 \%$ (see Table 3 and Fig. 5).

\subsection{Deformation of large pores V3, V6}

Surprisingly these pores underwent the lowest deformation after loading to $15 \%$. Pore V3 was deformed to $6.9 \%$ and pore V6 to $2.15 \%$. During and after unloading they did not recover but became even larger. The final irreversible deformation was $7.4 \%$ and $4.3 \%$, respectively (see Table 3 and Fig. 5).

In Fig. 5, the deformation of pores is given in percentage and the points are labeled with values in micrometer. Pores with widths of about 4-5 $\mu \mathrm{m}$ did not show some reversibility after deformation, even during unloading they continue to deform. A reason for such high deformation could be a higher degree of freedom of larger pores, they can rotate or rearrange the pore walls within the network. The deformation behavior is different due to various pore sizes leading to stress gradient in the sample. It leads to higher stresses on smaller pore walls and in turn to higher deformation of them. The larger pores deform slower and to a lesser extent. Apparently, irreversible deformations first occur in larger pores and then with higher loading also affect the smaller ones.

Based on these observations, a simple 2-d cell model similar to the one proposed by Rege et al. [22], where a damage evolution is accounted for, or the 3-d models by Gibson and Ashby [23] could be applied to carbon aerogels for modeling and characterization of their mechanical properties. Schwan et al. [17] presented a mathematical model to describe the flexibility of RF aerogels based on the idealization of the pore walls as simple bars undergoing bending and buckling. They further derived an expression for the flexibility as follows

$F=\frac{1}{E I_{w}} \propto \frac{1}{\rho_{e}^{2} t_{c}^{4}}$

where $E$ and $I_{w}$ are Young's modulus and the area moment of inertia, respectively. Further, $\rho_{e}$ and $t_{c}$ represent the envelope density and the size of cells, respectively. Accordingly, the lower the aerogel density, the larger the flexibility. Moreover, the smaller the pore size at a given envelope density, the larger the flexibility [17]. This phenomenon could be observed in the in-situ SEM analysis discussed above. The above conclusion holds not only for RF aerogels but also for carbon aerogels. Damage in the larger pore walls was observed to occur before the smaller ones. These observations have been echoed in the literature on silica aerogels describing their nanostructural damage [24]. There, the collapse of larger pores occuring sooner than the smaller ones was identified. This fits into the mathematical formulation discussed above since the damage mechanism based on buckling would result in the collapse of larger pore walls first. Note also that the connectivity of the aerogel network plays an important role in the mechanical structureproperty relationships. Based on the theory of Gibson and Ashby, for a perfectly connected structure, the power law exponent $m$ in the scaling relation $E \propto \rho^{m}$ should be always equal to 2 . For aerogels, this exponent varies typically between 2 and 4 . This is because the structure of aerogels is a result of a sequence of different processes, such as gelation, aging and shrinkage [25]. This results in a heterogeneous random connectivity of their network, making modeling of these aerogels from a micro-mechanical persepctive rather difficult. The above mentioned models are idealized within their given framework and certain mechanical quantities can only be estimated for non-cellular-shaped aerogel morphologies on the basis of these models. Nevertheless, they represent good starting points to correlate the kinematics at the microscale to the observations at the macroscale. Macroscopic mechanical characterization is presented in the following.

Uniaxial quasistatic compression tests were performed on s-CA, pCA, and sf-CA. Accordingly, s-CA exhibits brittle failure at very small compressive strains (around $2 \%$ - see Fig. 6a). The surface of the specimens is powdery and the progress of deformation results in a lot of particles/chunks of particles leaving the surface and falling out. This was one of the major challenges in getting accurate DIC measurements. To this end, several tests were conducted on specimens until satisfactorily reliable data was obtained. The results of the DIC are illustrated in Fig. 7b. The sudden brittle failure due to crack development and propagation is seen occurring at $1.9 \%$ compressive strain. Similar fracture behavior due to crack was observed during in-situ test (Fig. 3e). The empty pockets at the crack origin (and partly in the surroundings) are a result of the chunks of specimen falling apart upon fracture. Furthermore, under cyclic loading, one can observe hysteresis appearing even at very small strain values (see Fig. 6b). Other inelastic effects, such as cyclic stress softening and residual deformation can also be seen. The cyclic stress softening is not pronounced such that the reloading curve almost follows the unloading curve of the previous 

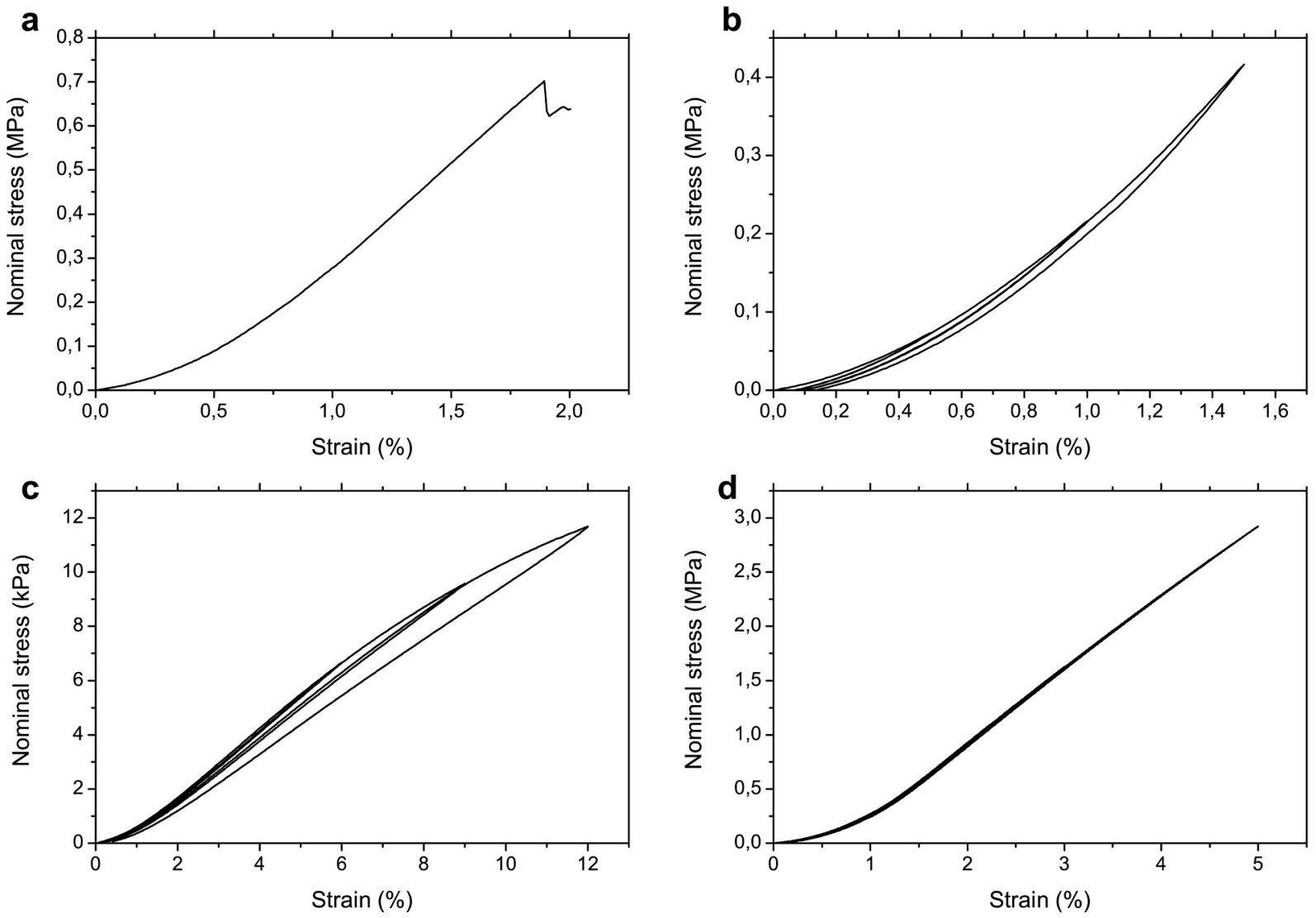

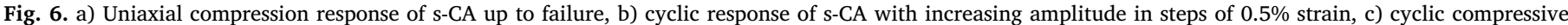
response of sf-CA with increasing amplitude in steps of $3 \%$ strain, d) cyclic compressive response of p-CA with increasing amplitude steps of $2.5 \%$ strain.

cycle. The aerogels also exhibit a good strain memory in a way that the stress-strain curve under reloading comes to the point of the maximum strain of the previous cycle and then follows the primary loading curve. The residual deformation (permanent set) appears to increase with increasing cyclic amplitude. After several tests, the following, almost linear, relation between the residual strain $\epsilon_{\text {res }}$ and the applied maximum strain $\epsilon_{\max }$ is proposed.

$\epsilon_{\text {res }} \propto \epsilon_{\max }^{0.8-1.1}$

This energy dissipated in a loading cycle $U_{\text {diss }}$ can be calculated as the area of the hysteresis loop and appears to increase quadratically in relation to $\epsilon_{\max }$ as

$U_{\text {diss }} \propto \epsilon_{\max }^{1.8-1.9}$.

Young's modulus of the s-CA aerogels is found to be around $45 \pm 5$ MPa.

Fig. $6 \mathrm{~d}$ shows step-wise increasing cyclic compression of p-CA. The two cycles can be hardly set apart, i.e., the unloading curve very closely follows the loading curve. This shows an almost elastic response up to $5 \%$ strain. p-CA fails at about $6 \%$ strain under compression. There is no observable residual deformation $(<0.1 \%)$ and a very negligible hysteresis. Young's modulus for the p-CA is found within the range of $55 \pm 5 \mathrm{MPa}$. Fig. 7a shows the DIC results of a p-CA specimen which failed at $3 \%$ compressive strain. The crack initiation along with propagation can be clearly seen in the displayed image.

Fig. $6 \mathrm{c}$ shows sf-CA with four loading-unloading cycles under compression. The hysteresis loop is seen to get wider with the increasing strain amplitude. Similar to p-CA, sf-CA shows no residual strains under compression of up to $15 \%$. Young's modulus for the sf-CA is found within the range of $0.07 \pm 0.01 \mathrm{MPa}$. Fig. 7c shows the DIC results of sf-CA (loading on the left, unloading on the right). The similarity in the loading and unloading curves can again be seen.

While carrying out tension tests on carbon aerogels is difficult, the three-point bending test appears feasible and useful. Its results are briefly reported below. Fig. 8 shows the force-deflection diagrams from the flexural tests. These data are then used to determine the maximal flexural stress and strain. The flexural stress is calculated as

$\sigma_{f}=\frac{3 F L}{2 b d^{2}}$,

where $F$ is the applied force and $b, d$ and $L$ are the width, depth and length of the support span, respectively. The flexural strain is calculated as

$\epsilon_{f}=\frac{6 \sigma d}{L^{2}}$,

where $\delta$ is the deflection at the center of the beam. The so obtained maximal flexural stresses and strains for p-CA, s-CA and f-CA aerogels are listed in Table 4, along with the above-mentioned Young's moduli.

Recalling our previous discussion on possible modeling strategies to describe carbon aerogels and correlating the in-situ microscopic results to macroscopic results portrayed above, the modeling strategies outlined by Gibson [26] can be well associated to the three different carbon aerogels. For example, s-CA exhibits brittle fracture at compressive strains of around $2 \%$. The microscopic collapse of the pore walls can be associated to brittle crushing. This is very much in contrast to sf-CA which show relatively higher flexibility and good recovery at moderate deformations. The collapse of the pore walls can be associated to their elastic buckling. Finally, p-CA shows excellent recovery in the linear elastic regime. The network failure could be associated to the plastic yielding or a combination of buckling and crushing. Interestingly, for all critical states mentioned above (elastic collapse stress, plastic collapse stress and brittle crushing strength) there exists a 
a

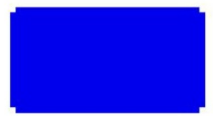

0.00

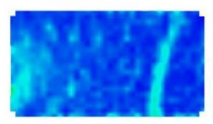

0.98

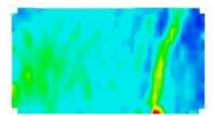

1.95

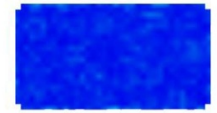

0.33

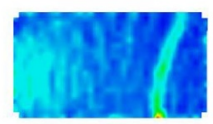

1.30

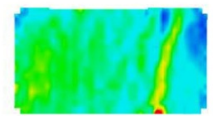

2.28

Engineering Strain [\%]

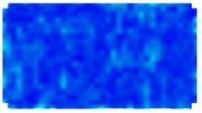

0.65

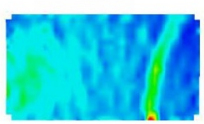

1.63

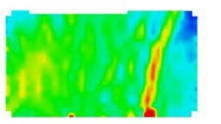

2.60
Loading Direction b

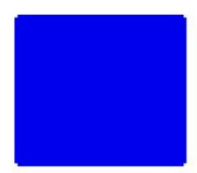

0.0

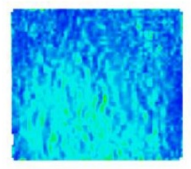

0.9

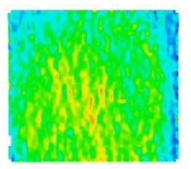

1.6

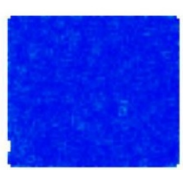

0.3

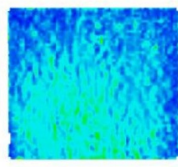

1.0

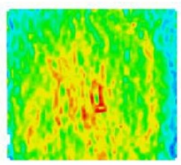

1.8

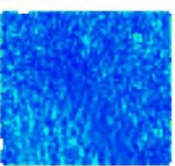

0.6

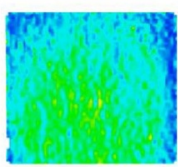

1.3

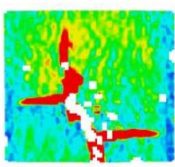

1.9
Engineering Strain [\%]

Loading Direction

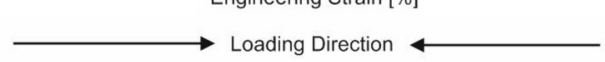

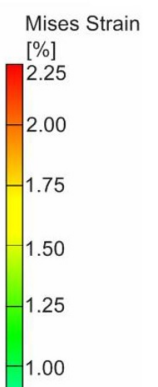

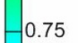

$-0.50$

$-0.25$

0.0

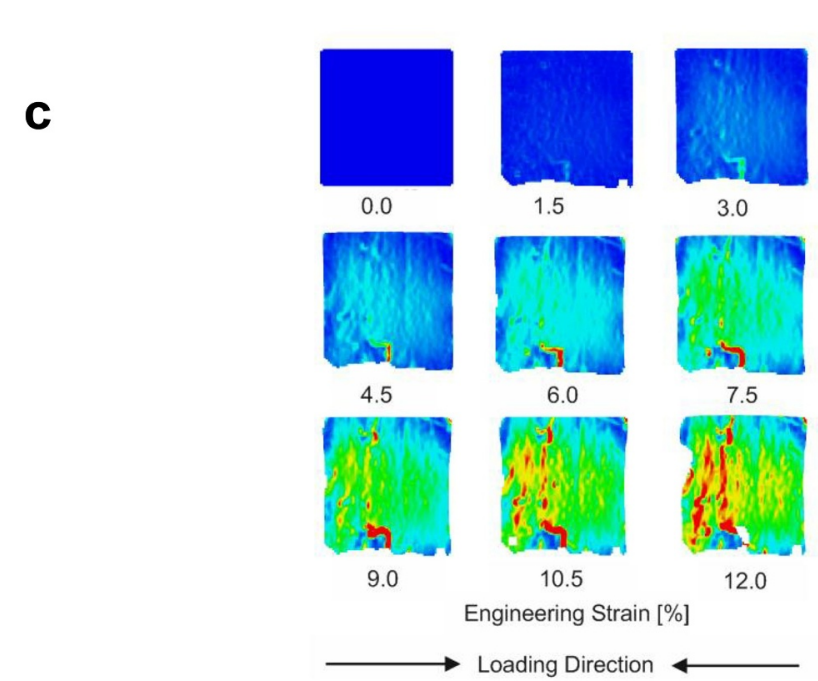

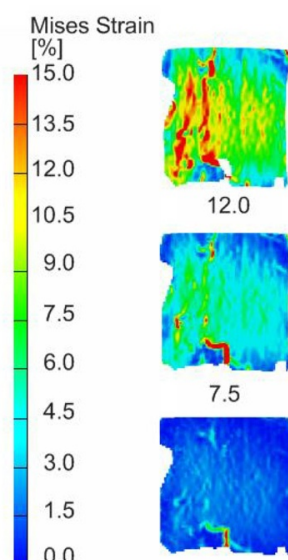

3.0

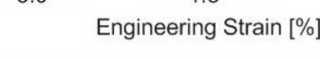

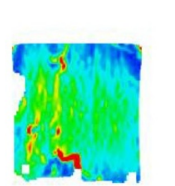

10.5

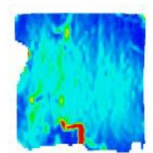

6.0

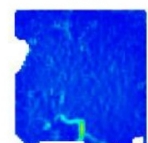

1.5

$\longleftrightarrow$ Unloading Direction $\longrightarrow$

9.0

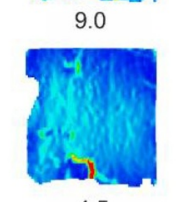

4.5

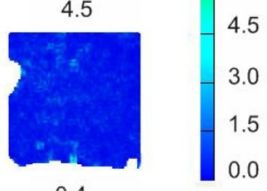

0.4

Fig. 7. a) DIC based deformation plots of p-CA up to $4 \%$ strain, b) DIC based deformation analysis via strain distribution of s-CA under uniaxial compression up to failure, c) DIC based strain distribution of sf-CA under uniaxial compression up to $12 \%$ strain.

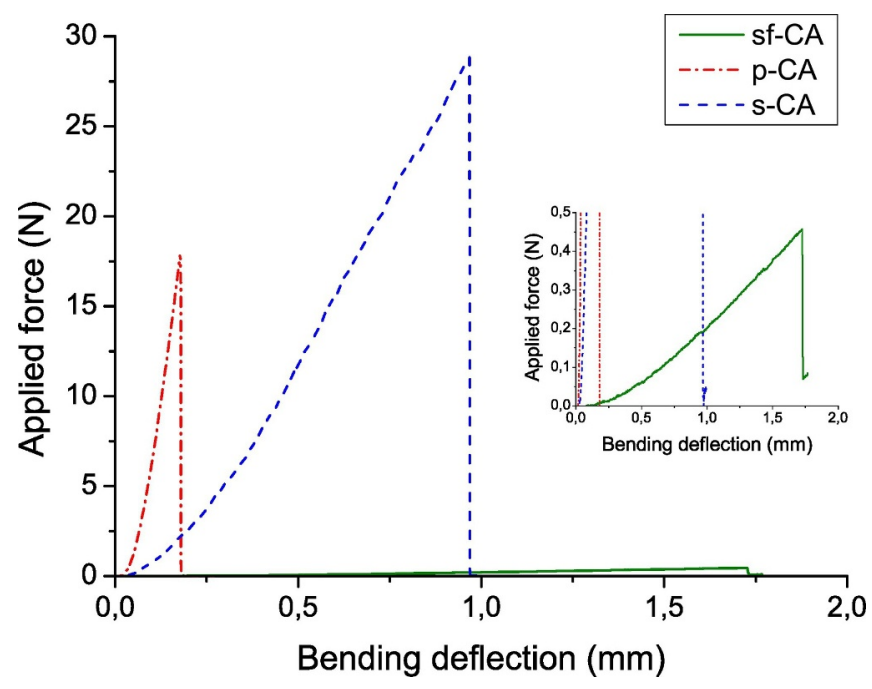

Fig. 8. Force $v s$. bending deflection diagram for sf-CA, p-CA and s-CA. The zoomed version of the same plot is separately presented for sf-CA in the inset.
Table 4

Mechanical properties of carbon aerogels $\left(\sigma_{f}, \epsilon_{f}\right.$ and $E$ denote the flexural stress, flexural strain and Young's modulus, respectively).

\begin{tabular}{llll}
\hline & $\sigma_{f}$ & $\epsilon_{f}$ & $E$ \\
\hline p-CA & $1.15 \pm 1.00 \mathrm{MPa}$ & $0.04 \pm 0.01$ & $55 \pm 5 \mathrm{MPa}$ \\
s-CA & $0.55 \pm 0.07 \mathrm{MPa}$ & $0.15 \pm 0.10$ & $45 \pm 5 \mathrm{MPa}$ \\
sf-CA & $6 \pm 2 \mathrm{kPa}$ & $0.440 \pm 0.005$ & $0.07 \pm 0.01 \mathrm{MPa}$ \\
\hline
\end{tabular}

power-law scaling with the relative densities. As an outlook to this work, an application of these mechanisms to characterize carbon aerogels would shed light to possible modeling approaches in the future. Determining the power-law scaling relations for all the three types of carbon aerogels with varying density would give insights into their network connectivity and similarities or dissimilarities with other kinds of aerogels.

\section{Conclusions}

In this paper, three types of carbon aerogels with different structural and mechanical properties have been investigated. Differences in the mechanical properties are linked to their microstructures. Quasistatic 
compression tests supported by digital image correlation are presented. The microstructure evolution under deformation is exhibited in the form of an in-situ study under SEM. The results show the fracture behavior of stiff carbon aerogel, where a disconnection between two particles could be observed under loading. A formation of crack was observed after the test in accordance with DIC measurement. The in-situ tests of flexible carbon aerogels reveal different deformation behavior of small and large pores. Large pores of about $4-5 \mu \mathrm{m}$ deform irreversibly. At the same time, deformation of smaller pores about $1 \mu \mathrm{m}$ is very high (23-24\%) and is almost reversible. These observations were proved with DIC. In this sense, the results of both methods are close to each other. p-CA appears to be the stiffest aerogel followed by s-CA and then sf-CA. p-CA shows nearly complete recovery until brittle fracture, which, to our best knowledge, has not been observed before. Similar brittle aerogels such as s-CA, for instance, or s-RF do not show such pronounced recovery and exhibit residual effects even at very low compressive strains. On the other hand, sf-CA shows complete recovery up to $15 \%$ compressive strains. Finally, flexural three-point bending tests are reported.

\section{Declaration of Competing Interest}

The authors declare that they have no known competing financial interests or personal relationships that could have appeared to influence the work reported in this paper.

\section{Acknowledgements}

The authors would like to thank Benjamin Ignatzi for support on carbonization.

\section{References}

[1] R.W. Pekala, Organic aerogels from the poycondensation of resorcinol with formaldehyde, J. Mater. Sci. 24 (1989) 3221-3227.

[2] R.W. Pekala, Melamine-formaldehyde Aerogels, (Feb. 4 1992).

[3] R.W. Pekala, Organic Carbon Aerogels from the Sol-gel Polymerization of PhenolicFurfural Mixtures, (Apr. 28 1998).

[4] L.W. Hrubesh, Aerogel applications, J. Non-Cryst. Solids 225 (1998) 335-342.

[5] R.W. Pekala, J.C. Farmer, C.T. Alviso, T.D. Tran, S.T. Mayer, J.M. Miller, B. Dunn, Carbon aerogels for electrochemical applications, J. Non-Cryst. Solids 225 (1998) 74-80.

[6] K. Balakumar, N. Kalaiselvi, High sulfur loaded carbon aerogel cathode for lithium-sulfur batteries, RSC Adv. 5 (43) (2015) 34008-34018.

[7] S.-W. Hwang, S.-H. Hyun, Capacitance control of carbon aerogel electrodes, J. NonCryst. Solids 347 (1-3) (2004) 238-245.

[8] W. Li, G. Reichenauer, J. Fricke, Carbon aerogels derived from cresol-resorcinol-formaldehyde for supercapacitors, J. Carbon 40 (15) (2002) 2955-2959.

[9] D.-W. Park, N.A. Canas, M. Schwan, B. Milow, L. Ratke, K.A. Friedrich, A dual mesopore c-aerogel electrode for a high energy density supercapacitor, J. Curr. Appl. Phys. 16 (6) (2016) 658-664.

[10] R. Saliger, U. Fischer, C. Herta, J. Fricke, High surface area carbon aerogels for supercapacitors, J. Non-Cryst. Solids 225 (1998) 81-85.

[11] L. Ratke, B. Milow, Aerogels for Foundry Applications, Springer, 2011, pp. 763-788.

[12] T. Woignier, J. Phalippou, Mechanical strength of silica aerogels, J. Non-Cryst. Solids 100 (1988) 404-408.

[13] T. Woignier, J. Primera, A. Alaoui, P. Etienne, F. Despestis, S. Calas-Etienne, Mechanical properties and brittle behavior of silica aerogels, Gels 1 (2) (2015) 256-275 https://doi.org/10.3390/gels1020256 https://www.ncbi.nlm.nih. gov/pubmed/30674176.

[14] K.E. Parmenter, F. Milstein, Mechanical properties of silica aerogels, J. Non-Cryst. Solids 223 (1998) 179-189.

[15] J.C.H. Wong, H. Kaymak, S. Brunner, M.M. Koebel, Mechanical properties of monolithic silica aerogels made from polyethoxydisiloxanes, Microporous Mesoporous Mater. 183 (2014) 23-29, https://doi.org/10.1016/j.micromeso.2013. 08.029 .

[16] M. Schwan, M. Naikade, D. Raabe, L. Ratke, From hard to rubber-like: mechanical properties of resorcinol-formaldehyde aerogels, J. Mater. Sci. 50 (16) (2015) 5482-5493 https://doi.org/10.1007/s10853-015-9094-x.

[17] M. Schwan, R. Tannert, L. Ratke, New soft and spongy resorcinol-formaldehyde aerogels, J. Supercrit. Fluids 107 (2016) 201-208, https://doi.org/10.1016/j. supflu.2015.09.010.

[18] A. Rege, M. Itskov, A microcell-based constitutive modeling of cellulose aerogels under tension, Acta Mech. 229 (2) (2018) 585-593 https://doi.org/10.1007/ s00707-017-1987-0.

[19] R. Saliger, V. Bock, R. Petricevic, T. Tillotson, S. Geis, J. Fricke, Carbon aerogels from dilute catalysis of resorcinol with formaldehyde, J. Non-Cryst. Solids 221 (2-3) (1997) 144-150.

[20] M. Schwan, L. Ratke, Flexible carbon aerogels, C 2 (3) (2016), p. 22 https://doi. $\operatorname{org} / 10.3390 / \mathrm{c} 2030022$.

[21] R.W. Pekala, C.T. Alviso, J.D. LeMay, Organic aerogels: microstructural dependence of mechanical properties in compression, J. Non-Cryst. Solids 125 (1990) 67-75.

[22] A. Rege, I. Preibisch, M. Schestakow, K. Ganesan, P. Gurikov, B. Milow, I. Smirnova, M. Itskov, Correlating synthesis parameters to morphological entities: predictive modeling of biopolymer aerogels, Materials 11 (9) (2018) 1670.

[23] L.J. Gibson, M.F. Ashby, Cellular Solids: Structure and Properties, Cambridge University Press, 1999.

[24] L. Perin, A. Faivre, S. Calas-Etienne, T. Woignier, Nanostructural damage associated with isostatic compression of silica aerogels, J. Non-Cryst. Solids 333 (1) (2004) 68-73.

[25] T. Woignier, J. Reynes, A.H. Alaoui, I. Beurroies, J. Phalippou, Different kinds of structure in aerogels: relationships with the mechanical properties, J. Non-Cryst. Solids 241 (1) (1998) 45-52.

[26] L.J. Gibson, Biomechanics of cellular solids, J. Biomech. 38 (3) (2005) 377-399. 